\title{
Clinical profile and outcome of acute encephalitis syndrome (AES) patients treated in College of Medical Sciences-Teaching Hospital
}

\author{
Thapa L, ${ }^{1}$ Twayana RS, ${ }^{2}$ Shilpakar R, ${ }^{3}$ Ghimire MR , ${ }^{4}$ Shrestha A, ${ }^{5}$ Sapkota $S,{ }^{6}$ Rana PVS \\ ${ }^{1}$ Assistant Professor, ${ }^{6}$ Staff Nurse, ${ }^{7}$ Professor, Department of Neurology, College of Medical Sciences, \\ Bharatpur, Nepal. \\ ${ }^{2}$ Lecturer, Department of Internal Medicine, Kathmandu University Medical College, Dhulikhel, Nepal \\ ${ }^{3} \mathrm{MD}$ Resident,Department of Internal Medicine, College of Medical Sciences, Bharatpur, Nepal. \\ ${ }^{4} \mathrm{MD}$ Resident, Department of Internal Medicine, National Medical Sciences, Birgunj, Nepal \\ ${ }^{5}$ Medical officer, Nuwakot (Trisuli) district hospital, Nuwakot, Nepal
}

\section{ABSTRACT Objective}

Acute encephalitis syndrome is a cause of significant morbidity and mortality in Nepal. Although Japanese encephalitis virus (JEV) was thought to be a major cause for acute encephalitis syndrome, more non-Japanese encephalitis virus cases are reported. The outcome of patients with acute encephalitis syndrome is variable. Our study was designed to study the clinical profile and outcome of patients with acute encephalitis syndrome managed in tertiary care center in central Nepal.

\section{Methods}

The record of patients admitted with diagnosis of acute encephalitis syndrome,from January 2010 to December 2010 in College of Medical Sciences-Teaching Hospital (CMS-TH) was reviewed. They were classified clinically as meningitis, encephalitis and meningoencephalitis. The clinical details and reports of the patients were recorded and analyzed.

\section{Results}

Total of 85 cases of meningitis and encephalitis were identified. Mean age was 19.18 years. Fifty-six (65.9\%) patients were males and 29 (34.1\%) were females. Sixty (70.58\%) patients had meningitis, 8 (9.41\%) had encephalitis, and $17(20.0 \%)$ had meningoencephalitis. JE serology was positive in 4 patients (4.7\%). Seventy-two (84.7\%) patients made full recovery and were discharged from hospital. Thirteen (15.3\%) patients left against medical advice (LAMA).

\section{Conclusion}

Acute encephalitis syndrome is still a major public health problem in Nepal. Few of these patients have Japanese Encephalitis. There is a trend towards improved outcome because of availability of improved health services. However, financial constraint remains a challenge in management of acute encephalitis syndrome.

KEY WORDS: Acute encephalitis syndrome, encephalitis, Japanese encephalitis, meningitis, mening oencephalitis. 
Journal of College of Medical Sciences-Nepal, 2013, Vol-9, No-2,

\section{INTRODUCTION}

Acute encephalitis syndrome (AES) is defined as acute onset of fever and a change in mental status (including symptoms such as confusion, disorientation, coma, or inability to talk) and/or new onset of seizures (excluding simple febrile seizures) in a person of any age at any time of year. ${ }^{1}$ Japanese encephalitis (JE), a leading cause of AES in our continent, ${ }^{2}$ affects about 50,000 people and causes 10,000 deaths each year. ${ }^{1}$ However, because of incomplete surveillance in many affected areas, reported cases of JE greatly under-represent the true impact. ${ }^{1}$ Even though to control JE, the identification of patients with AES is essential, AES case definition for children on hospital admission had only $65 \%$ sensitivity for JEV-infected cases. ${ }^{3}$ The first outbreak of JE in Nepal was reported in 1978 from Rupandehi, a terai district. ${ }^{4}$ Outbreak of JE in the hilly region(Kathmandu valley) was confirmed in $1997,{ }^{5}$ and in 2006 JE endemicity in Kathmandu was established. ${ }^{6}$ Total 24 Terai districts are known to be endemic (Figure: 1) with case fatality rate (CFR) ranging from 5-29\% (average 10\%). Average case fatality in all ages is about $20 \%$ in Nepal. ${ }^{4}$ The landscape of AES in India has changed in the previous decade, and both outbreak investigations and surveillance studies have increasingly reported non-JEV aetiologies. ${ }^{7}$ Our study was designed to describe the clinical profile of AES and understand their clinical outcome.

\section{Japanese encephalitis endemic districts-Nepal}

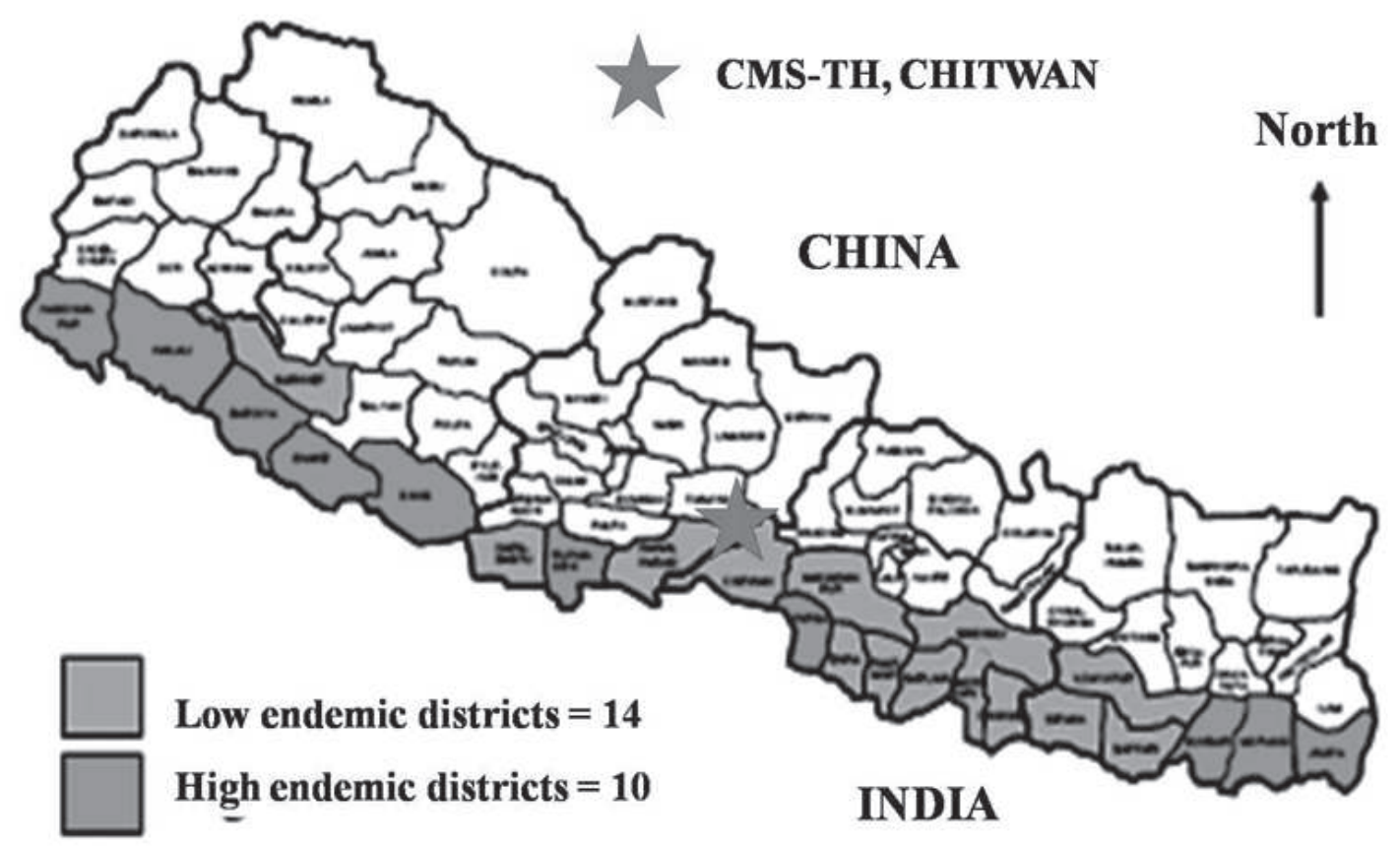

Figure 1: Terai districts of Nepal. CMS-TH location (Star) showing its broad catchment area

\section{METHODS}

This is a descriptive, cross-sectional study of patients admitted from January 2010 to December 2010 with diagnosis of AES in CMS-TH, a tertiary care neurological center in Chitwan. Records of all the patients having features of AES as WHO case definition criteria were included in the study. Clinically they were classified as: (a) meningitis, if they had fever with meningeal signs and absence of seizure, (b) encephalitis, if they had fever with 
Thapa LJ et al. Clinical profile and outcome of acute encephalitis

seizure but absence of meningeal signs, and (c) meningoencephalitis, if patients had fever, seizure and altered sensorium. Clinical details and investigations particularly JE serology (ELISA for $\operatorname{IgM}$ ) and their clinical outcome were recorded and analyzed. The results of JE serology was obtained from Nepal Public Health Laboratory, Teku Kathmandu through the local government agency.

\section{RESULTS}

Total 85 cases of AES were admitted from the January 2010 to December 2010. Males were more than females [56 (65.9\%): $29(34.1 \%)]$. Age ranged from 0.08 year to 86 years (Mean age: 19.18 years). Forty-nine $(57.6 \%)$ patients were from Chitwan district. Others were from neighboring districts: 30 (35.3\%) from Makwanpur, 5 (5.9\%) from Bara and $1(1.2 \%)$ from Parsa. Clinical features included fever in $82(96.5 \%)$ patients, unconsciousness in $44(51.8 \%)$, headache in $41(48.2 \%)$, convulsion in $41(48.2 \%)$, disorientation in $38(44.7 \%)$, loss of coordination in 24 (28.3\%) patients.(Figure: 2$)$

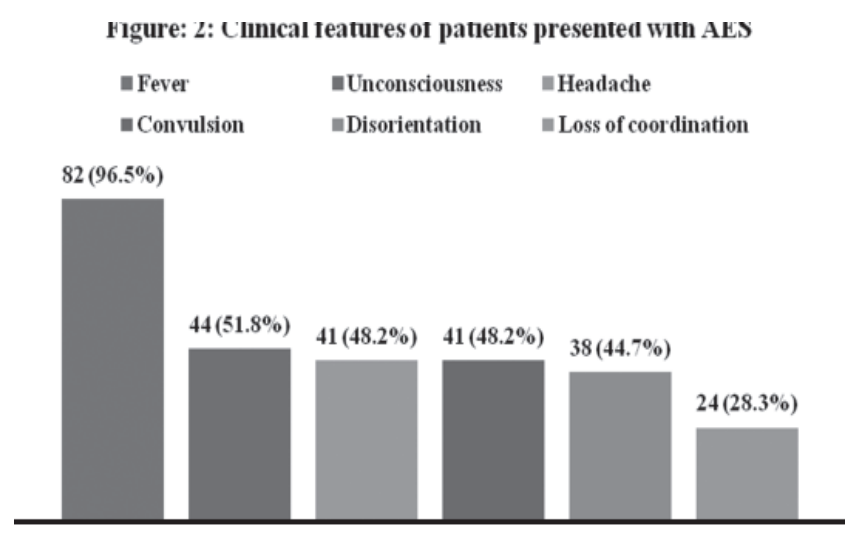

Fifty-nine $(69.41 \%)$ cases had presented with clinical meningitis out of which $40(47.05 \%)$ were males and 19 (22.35\%) were females. Similarly, $17(20.0 \%)$ cases presented with meningoencephalitis, out of which $9(10.58 \%)$ were males and $8(9.41 \%)$ were females. Eight $(9.41 \%)$ patients presented with encephalitis, $6(7.05 \%)$ males and $2(2.35 \%)$ females. $1(1.17 \%)$ patient was diagnosed to have TB meningitis. (Table 1)

\section{Table 1: Patterns of the distribution of cases $(n=85)$}

\begin{tabular}{l|c|c|c} 
Diagnosis & Male & Female & Total \\
Meningitis & $40(47.05 \%)$ & $19(22.35 \%)$ & $59(69.41 \%)$ \\
Meningo & $9(10.58 \%)$ & $8(9.41 \%)$ & $17(20.0 \%)$ \\
encephalitis & & & \\
Encephalitis & $6(7.05 \%)$ & $2(2.35 \%)$ & $8(9.41 \%)$ \\
TB & $1(1.17 \%)$ & $0(0 \%)$ & $1(1.17 \%)$ \\
Meningitis & & &
\end{tabular}

Serology for JE had been tested from CSF in 4 $(4.7 \%)$ patients, serum in $45(52.9 \%)$ patients and both serum and CSF in $36(42.4 \%)$ patients. Out of 85 cases $4(4.70 \%)$ were JE serology $(\operatorname{IgM})$ positive: 2 patients $(50 \%)$ from Chitwan and 2 (50\%) neonates from Makwanpur.

Seventy-two $(84.7 \%)$ patients made full recovery and were discharged from the hospital. Thirteen $(15.3 \%)$ patients left against medical advice (LAMA) because of financial constraints.

Fifty-one $(86.44 \%)$ out of 59 patients with meningitis made full recovery. Similarly, 13 (76.47\%) out of 17 patients of meningoencephalitis recovered and all (100\%) of patients with encephalitis made full recover and discharged. Nine patients $(15.25 \%)$ of meningitis and 4 patients (23.52\%) of meningoencephalitis patients left against medical advice due to financial reasons.(Table 2) 
Table 2: Outcomes of the cases $(n=85)$

\begin{tabular}{lcc|c|}
\hline Diagnosis & Discharged & LAMA & Total \\
\hline Meningitis & $50(84.74 \%)$ & $9(15.25 \%)$ & $59(100 \%)$ \\
Meningoencephalitis & $13(76.47 \%)$ & $4(23.52 \%)$ & $17(100 \%)$ \\
Encephalitis & $8(100 \%)$ & $0(0 \%)$ & $8(100 \%)$ \\
TB Meningitis & $1(100 \%)$ & $0(0 \%)$ & $1(100 \%)$ \\
Total & $72(84.7 \%)$ & $13(15.29 \%)$ & $85(100 \%)$
\end{tabular}

\section{DISCUSSION}

Our study showed that AES affected entire age group from children to elderly patients. Although children are more vulnerable, a person of any age, at any time of a year is known to be affected by AES although there is tendency for high incidence specifically during monsoon and post monsoon period. ${ }^{8,9,10,11}$

$\mathrm{JE}$ is one of the important causes of AES. Although reports emphasize JE in pediatric populations, JE also produce morbidity and mortality in adults in Nepal. $^{12}$

Vaccination has brought a marked difference in incidence of JE. Hoke et al have demonstrated this difference; 51 per 100,000 in vaccinated when compared to 5 per 100,000 in non-vaccinated. ${ }^{13}$ Vaccination campaigns with SA-14-14-2 vaccine are although protecting the children less than 15 years, older people are more affected. ${ }^{14}$ To note, none of our patients were vaccinated against JE.

Males were more affected in our cohort. Similar pattern was observed in surveillance in Bangladesh where amongst 218 patients, $66 \%$ of the affected were males. ${ }^{11}$
Kakkar et al and Thomas et al also demonstrated males to be affected more than females. ${ }^{8,12}$

Interestingly, Roy et al have postulated that the male dominance may be due to the reason of males sleeping outside the houses with bare body and hence more prone for mosquito bite. ${ }^{15}$ However, exact reason for male dominance in AES still remains elusive.

Majority (57.6\%) of our patients were from Chitwan, a terai district. Terai districts in Nepal are known to be "hot spot" for JE. In 1978, the first outbreak of JE in Nepal was documented from Rupandehi, a terai district. ${ }^{4} \mathrm{JE}$ recently is observed to affect hilly region as documented by identification of JE in Kathmandu valley in 1997 ${ }^{16}$ and $2006 .{ }^{17}$

By now we know that JE is not uniformly distributed in our country, but is found to be primarily confined to 24 districts of Terai that border India. ${ }^{12}$ Although AES is not synonymous with JE, the spatial, temporal, and landcover configuration distributions of JE and AES has been observed to have a high similarity in pattern between JE and AES across all three dimensions 
Thapa LJ et al. Clinical profile and outcome of acute encephalitis

of comparison. ${ }^{18}$ However, we did not have any patients from hilly regions.

The major clinical presentations of AES in our study were fever, unconsciousness, headache, convulsion, disorientation and lack of coordination in decreasing frequency. All of these features plus ocular palsies, hemiplegia, extrapyramidal dysfunctions have been documented in various studies. ${ }^{15,16,17,19}$ Early clinical findings suggested in AES are an increase in irritability, somnolence or abnormal behavior greater than that seen with usual febrile illness. ${ }^{9}$

Amongst the clinical subtypes, clinical meningitis was the major presentation followed by meningoencephalitis and encephalitis. Intriguingly, males predominated throughout entire clinical subtypes. One patient was diagnosed as TB meningitis. Serology for JE ( $\operatorname{IgM})$ was tested in all 85 cases and four patients $(4.07 \%)$ were found to be positive. This low positivity for JE is not unusual in AES because JE once thought to be the major identified cause of AES is however, not synonymous to AES. AES is associated with a variety of other pathogens including other viruses, bacteria and parasites. It has been estimated that a quarter to one-third of AES cases in Nepal are due to JE. ${ }^{12,20}$ At this point it has to be however understood that an Indonesian study with special attention to testing protocol detected $30 \%$ more JE infections ${ }^{21}$ but a study from Nepal, after increasing the case load by $30 \%$ from originally detected JE cases, still found a significant number of JE negative cases presenting as AES. ${ }^{12}$ In a study from Japan, among 105 aetiologically diagnosed cases of AES, the most common causative agents were measles virus (23\%), rubella virus (23\%) and HSV
(20\%). ${ }^{22}$ After carefully observing the clinical pattern and reviewing literatures, we are convinced that we also have causes other than JEV that cause AES in our set-up but because of limited resources, we are not able to test for different etiologies.

Outcome in AES is variable in different studies especially in JE positive and JE negative cases. Some study demonstrated similar deaths ${ }^{12}$ while other emphasizes AES due to JE to have better outcome. ${ }^{20}$ To note, Thomas et al have demonstrated higher mortality in adults over 35 years. ${ }^{12}$ Majority $(84.7 \%$; $n=72)$ of our patients made full recovery and were discharged. All our patients with JE made full recovery, although the number of patients was less. However, similar results are less likely to be obtained in a large scale study. But better services and management protocols followed in our institution definitely have an influence on better outcome of our patients. We did not find the record of neurologic sequelae of our patients at discharge.

Leaving against medical advice (LAMA) is usual in developing countries like ours where we do not have proper health policy and where patients have to pay for the treatment. Thirteen $(15.3 \%)$ patients left against medical advice (LAMA) because of financial constraints. Similar trend is also reported from our neighboring country. ${ }^{23}$

\section{LIMITATIONS}

Our study has an inherent limitation of a retrospective study. In addition we were unable to retrieve lab data and describe CSF profiles of all patients. Because of lack of lab support we were not able to get other virological profile, which may have identified other causes of AES. 
Journal of College of Medical Sciences-Nepal, 2013, Vol-9, No-2,

\section{CONCLUSION}

AES is a major burden in our clinical practice. NonJE causes for AES should be strongly considered as there is documentation of other etiologic agents. Better treatment facility and protocols may have better outcome in these patients. Financial constraint remains a major challenge in management.

\section{REFERENCES}

\section{Japanese encephalitis surveillance} standards.[Internet] 2006 [cited 2013 August

30]; Available from: http://www.path.org/files/ WHO_surveillance_standards_JE.pdf

2. Rao PN. Japanese Encephalitis. Indian Pediatrics 2001;38:1252-64.

3. Solomon T, Thao TT, Lewthwaite P et al. A cohort study to assess the new WHO Japanese Encephalitis surveillance standards. Bulletin of the World Health Organization 2008;86:178-86.

4. Bista MB, Shrestha JM. Epidemiological situation of Japanese encephalitis in Nepal. $J$ Nepal Med Assoc. 2005;44:51-6.

5. Zimmerman MD, Scott RM, Vaughn DW et al. Short report: an outbreak of Japanese encephalitis in Kathmandu, Nepal. Am J Trop Med Hyg. 1997;57:283-4.

6. Partridge J, Ghimire P, Sedai T et al. Endemic Japanese encephalitis in the Kathmandu Valley, Nepal. Am JTrop Med Hyg. 2007;77:1146-9.

7. Joshi R, Kalantari SP, Reingold A et al. Changing landscape of acute encephalitis syndrome in India:A systematic review. Natl Med J India 2012;25:212-20.
8. Kakkar M, Rogawski ET, Abbas SS et al. Acute encephalitis syndrome surveillance, Kushinagar District, Uttar Pradesh, India, 2011-2012. Emerg Infect Dis. [Internet] 2013 [cited: 2013 August 30]; Available from: http://dx.doi.org/ 10.3201/eid1909.121855

9. Khinchi YR, Kumar A, Yadav S. Study of acute encephalitis syndrome in children. Journal of College of Medical Sciences-Nepal 2010;6:7-13.

10. Jmor F, Emsley H, Fischer $M$ et al. The incidence of acute encephalitis syndrome in Western industrialised and tropical countries. Virology Journal 2008;5:134.

11. Surveillance for encephalitis in Bangladesh: preliminary results. [cited 2013 August 30]; Available from: http://www.icddrb.org/whatwe-do/publications/cat_view/52-publications/ 10042-icddrb-periodicals/10048-health-andscience-bulletin-bangla-and-english/10600-vol2-no-4-english-2004/11850-surveillance-forencephalitis-in-bangladesh-preliminary-results.

12. Wierzba TF, Ghimire P, Malla $S$ et al. Laboratory-based Japanese Encephalitis Surveillance in Nepal and the Implications for a National Immunization Strategy. Am. J. Trop. Med. Hyg., 2008;78:1002-6.

13. Hoke CH, Nisalak A, Sangawhipa Net al. Protection against Japanese encephalitis by inactivated vaccines. $N$ Engl J Med 1988;319:608-14.

14. Borthakur AK, Das N, Bora BJ. Data from the World Health Organization (WHO) National Network Laboratory for Japanese Encephalitis. J Glob Infect Dis. 2013;5:76-9. 
Thapa LJ et al. Clinical profile and outcome of acute encephalitis

15. Roy A, Tandon R, Agarwal SK. Seroprevalence of Japanese encephalitis virus infection in Lucknow, Uttar Pradesh. Indian J Med Res 2006;124:211-12.

16. Zimmerman MD, Scott RM, Vaughn DW et al. Short report: an outbreak of Japanese encephalitis in Kathmandu, Nepal. Am J Trop Med Hyg. 1997;57:283-4.

17. Partridge J, Ghimire P, Sedai T et al. Endemic Japanese encephalitis in the Kathmandu Valley, Nepal. Am JTrop Med Hyg. 2007;77:1146-9.

18. Robertson C, Pant DK, Joshi DD et al. Comparative Spatial Dynamics of Japanese Encephalitis and Acute Encephalitis Syndrome in Nepal. PLoS ONE 2013;8:e66168.

19. Clinical management of acute encephalitis syndrome including Japanese encephalitis. [Internet] 2009 [cited 2013 August 31]
Available from: http://nvbdcp.gov.in/Doc/ Revised\%20guidelines\%20on\%20AES_JE.pdf

20. Rayamajhi A, Singh R, Prasad R et al. Study of Japanese encephalitis and other viral encephalitis in Nepali children. Pediatr Int 2007;49:978-84.

21. Kari K, Liu W, Gautama K et al. A hospitalbased surveillance for Japanese encephalitis in Bali, Indonesia. BMC Med 2006;4:8.

22. Kamei S, Takasu T: Nationwide survey of the annual prevalence of viral and other neurological infections in Japanese inpatients. Intern Med 2000;39:894-900.

23. Sarkari NB, Thacker AK, Barthwal SP et al. Japanese encephalitis (JE). Part I: clinical profile of 1,282 adult acute cases of four epidemics. J Neurol. 2012;259:47-57. 